\title{
Energy Efficiency Performance for 5G Cellular Networks
}

\author{
Afef Bohli and Ridha Bouallegue \\ University of Tunis El Manar, \\ Engineering School of Tunis \\ \{afef.bohli, ridha. bouallague\}@gmail.com
}

\begin{abstract}
The next generation of wireless connectivity-the fifth generation, or 5G will show an intense demand for data rates. Densified heterogeneous cellular networks, where a large number of small cell stations is deployed on traditional cellular topologies, is defined as one among the most important solutions in order to sustain both data volume and capacity demands. However, energy consumption in such network deployment goes up in proportion along with the rise of small cell station numbers. Thus, enhancing Energy Efficiency (EE) becomes an important aim to implement future wireless mobile networks. In this paper, a dynamic spectrum access scheme is suggested in an ultra-dense heterogeneous network based on a cognitive monitor sensing strategy to improve the EE while meeting the required capacity. The EE analytical expression is derived based on the stochastic geometry theory. Through the simulation results, the performance of the suggested scheme are conducted.
\end{abstract}

Keywords: Dense heterogeneous networks; spectrum efficiency; common channel protocol; inter-cell-interferences; cognitive radio; stochastic geometry; coverage probability.

\section{Introduction}

The growth of communication traffic from $50 \%$ to $100 \%$ will contribute to an increase in the energy consumption in the communication networks [1]. Given that information and communication technologies systems and devices account for about $2 \%$ of global CO2 emissions [2][3] and this contribution is expected to increase to $4 \%$ by the year 2020 [4], energy consumption will be an important economic cost factor for the Ultra-Dense Heterogeneous Network (UDHetNet). According to some studies, the main source of energy consumption in the mobile network is the base stations. In fact, the base station is the most energy-intensive element, given that $80 \%$ [5] of the mobile networks $\mathrm{CO} 2$ contribution came from the base stations.

Therefore, and given the swelling in the number of cell stations in the UDHetNet, future communications will experience a crucial challenge introduced under the slogan of green network aiming to reduce the $\mathrm{CO} 2$ emission.

To this end, future networks based on the UDHetNet deployment must be designed to be as energy efficient as possible while keeping in mind the principle spectrum efficiency.

Various aspects for saving energy in the communication systems, such as energy efficient wireless transmission technologies, interference mitigation concepts, network architecture, protocols and opportunistic cognitive spectrum-sharing schemes, are under consideration.

Given that, the higher power consumption portions were related to the circuits of the active base stations, [6]suggested a base station's deactivation technique. Accordingly, deactivation of the non-utilized base stations was evaluated as a promising approach for reducing the energy consumption in mobile networks. The simulation results prove a significant improvement in terms of energy efficiency compared to the traditional sleeping mode. Nevertheless, under the consideration of a multiple input, multiple output-orthogonal frequency division multiplexing small cells network, the Inter-Cell-Interferences (ICIs) are neglected assuming a non-overlapped sharing carrier.

In order to reduce the amount of energy consumption in a dense cell station (considered as access points), [7] put forward the on-demand resource methods strategy. This technique allowed switching, dynamically, on or off, the stations according to their number of users, which was based on the loaded traffic volume as well the set of active users in the coverage zone. A green cluster was conceived based on a set of nearby cell stations. These stations recognized themselves through the use of controlled beacons. Although the use of this strategy improved energy efficiency, it faced a trade-off between service quality and saved power. As the cell sizes were large, the overall cluster coverage was bigger, resulting in higher energy savings but at expense of lower quality service.

A solution based on the dynamic adjustment of the size of cell station coverage area was proposed in [8]. Three parameters, the estimated user demands, traffic load and channel conditions, were utilized for cell station size adaptation. Both distributed and centralized algorithms were conducted. In each case of them, the cell zone service could be implemented in the central station or distributed in all stations, respectively. The main function of this service was to detect and collect information relative to the three defined parameters. Once done, a decision 
about the cell zooming concept was conducted. Cooperation techniques and relaying methods were considered in the design of this strategy. This concept would provide benefits in terms of energy efficiency by saving the consumed power. Yet, it was challenging to estimate the traffic load, given the significant variations of traffic volume due to fluctuations.

A user's association and power allocation concept to maximize the energy efficiency metric in a two-tier heterogeneous uplink network was proposed in [9]. Unlike the classical mechanism that would allocate the rate to the subcarrier based on an equal manner, this method attributed the highest minimum rate to the subcarrier with the highest channel-to-noise ratio. The numerical results indicated that the rate-proportional method considerably enhanced the energy efficiency.

However, given the dense cell station deployment, the user association in a future UDHetNet will face additional challenges related to the interference management.

It is agreed that the conventional Inter-Cell-Interference-Coordination (ICIC) techniques - defined in the LTE $8 / 9$ releases [10] and aiming to control the ICI through the use of the radio resource management approaches and the enhanced ICIC - introduced in the LTE R10 [11] and aiming to coordinate the transmissions of multiple cell stations in the frequency and time domains based on the on-power control mechanisms using Almost Blank Subframes (ABS) - are not considered as fully effective solutions in UDHetNets. This, result, first, from the nature of these networks which are arbitrarily planned and dynamically deployed [12]. And second, from the fact that, when all the small cells share the same ABS and are so closely, intra-cell-interferences become a serious problem. A dynamic ABS for each small stations, is proposed by some previous studies, to avoid this type of intra cell interference [13][14][15]. Yet, in an ultra-dense heterogeneous network, keeping the macro base station silent during all the ABS that are accorded dynamically to various small cell stations bringing about the reduction of the allocated macro-users resources for data transmission, yielding in a spectrum inefficiency.

Thus, the problem of interference is addressed by [16] which coped with the problem of co/cross-tiers ICI issue in the heterogenous network through the use of the cognitive radio concept. The Small Cell Stations (SCSs) presented the secondary system and the macro base station were the primary system. Each SCS senses the free channels of the MBS and the remaining SCSs to serve their users. A semi and full cognitive models are assumed, where in the first case only a perfect MBSs sensing is assumed. However in the second case, in addition to the previous assumption, the perfect SCSs sensing is also considered. Although this technique reduces the interference, it does not reflect the realistic network state given that, in an unplanned SCSs deployment, the miss detection probability should be taken into account. [17] took the same previous issue considering an imperfect SCSs sensing model. However, the case of non existence (perfect or imperfect) sensing idle channels, the SCSs will not have spectrum resources to serve their users.

In our previous work, [18] a dynamic spectrum access scheme, was suggested, to cope with the co/cross ICI in a two-tier heterogeneous network and improve the energy efficiency. Based on the cognitive radio approach with a novel sensing strategy [19], the amount of interference was reduced compared to other traditional scenarios.

This paper presents an extension of our previous work [18]. Thus, an interference mitigation scheme in an ultradense heterogeneous network is conceived leading to improve the energy efficiency while guaranteing a required throughput. The rest of this paper is organized as follows. First, in section II, the system model is described and the suggested protocol is defined. Then, in section III, the coverage probability expression is derived, based on the stochastic geometry model. in Section IV the Energy Efficiency (EE) formula is derived. After that, the performance of the suggested framework is quantified and proved, in section $\mathrm{V}$, through the numerical results. Finally, the paper is concluded in section VI.

\section{System Model and assumption}

We consider an UDHetNet consisting of independent $K$ tiers of randomly located SCSs, which differ, across tiers, in terms of spatial densities and transmit powers. We assume that the SCSs belonging the $i^{\text {th }}$ tier, with $i \in\{K\}$ and $K \geq 1$, follow a stationary independent marked poisson point process (i.m.p.p.p) of a spatial density $\lambda_{i}$. These SCSs have the same transmit power $P_{R S_{i}}$, the same static power $P_{S S_{i}}$ and the same SINR threshold $\widetilde{S I N R}{ }_{i}$. To simplify the derivation of the coverage probability expression, we assume that $\widetilde{\operatorname{SINR}} i>1(0 d B)[20]$. In fact, using this assumption, [20] proved that at most one SCS in the entire network could provide a SINR greater than the required threshold. Furthermore, and motivated by the same reference, the thermal noise was neglected given that it had a very limited effect on the coverage probability. A Rayleigh fading model is assumed with a gain channel $h$ that follows an exponential distribution with a mean $\eta=1$. In addition, an omnidirectional path-loss model $l(|x|)=x^{\alpha}$ is adopted, where $\alpha$ presents the path loss exponent. We consider that for each tier a set of $M_{i}$ sub-channel are assigned and that the $\bigcap_{i \in\{K\}, k>1} M_{i} \neq \emptyset$. We note $p_{\text {coll }_{i}}$ the collision probability defining the fact that a subset of the $M_{i}$ sub-channel is utilized simultaneously by some SCSs (either in the same tier or 
the other tiers). Let us assume that this set of SCSs follows an i.m.p.p.p, which is a thinning of the i.m.p.p.p $\phi_{i}$ of the intensity measure $\lambda_{i}$ with the retention probability $p_{\text {coll }_{i}}$, yielding an i.m.p.p.p of the intensity measure $\xi_{i}=p_{\text {coll }_{i}} \lambda_{i}$. Moreover, we assume $\zeta \in[0,1]$ the probability that beacons are broadcasted and complementally $1-\zeta$ the probability that the SCSs are not sending beacons. Therefore, using the thinning method [21], the number of beacons $N_{i}$ broadcasted by the SCSs will follow an i.m.p.p.p of an intensity measure $\Xi_{i}=\zeta \lambda_{i}$ and will be provided by the probability $P_{N_{i}}=\frac{(A \Xi)^{N_{i}}}{N_{i} !} \exp \left(-A_{i} \Xi_{i}\right)$, where $A_{i}$ is the $\mathrm{i}^{\text {th }}$ SCS considered area.

Furthermore, the DCCMC (Dynamic Common Cognitive Monitor Channel) MAC layer protocol, proposed and described in [18], is assumed, which means that, each station, after measuring the holding time (vacant channel time) distribution of each idle channel [22], selects the one with the highest maximum probability density function value as the common control channel DCCMC and broadcasts this information as beacons through all the detected idle channel. The user, which is listening on the idle channels, once received the broadcasted beacons, will select the one with the maximum SINR and adapts its transmission parameters in order to switch into this channel.

\subsection{Coverage probability}

The coverage probability for a typical user in the K-tiers heterogenous network, based on the DCCMC concept, represents the Complementary Cumulative Distribution Function (CCDF) of the SINR distribution reached from the best selected DCCMC, which is given as follows [20]:

$$
\operatorname{SINR}_{x_{i}}=\frac{P_{i} h x_{i}^{-\alpha}}{\sum_{k=1}^{K} I_{x_{k_{(k \neq i)}}}}
$$

where $x_{i}$ is the users position, $I_{x_{k}(k \neq i)}$ is the noise term coming from the aggregate inter-tier interferences from the $k^{\text {th }}$ tier .

Setting $M_{i}$ as the assigned carrier for the $i^{\text {th }}$ tier, a typical user $\mathrm{UE}_{i}$, linked to a station from the $i^{\text {th }}$ tier will suffer from the interferences coming from the set of the stations from the same tier as well the other tiers that use some of the $M_{i}$ allocations resources of its tagged station.

Let take $p_{\mathrm{coll}_{i}}$ as the probability of collision given as follows:

$$
p_{\text {coll }_{i}}=1-\prod_{j=1}^{K}\left(1-\frac{1}{M_{i}}\right)^{M_{j}}
$$

In fact, the probability of collision, defining the event that "At least one subcarrier from $M_{i}$ is used simultaneously by the other tiers" is formulated as follows:

$$
\begin{aligned}
& \left.p_{\text {coll }_{i}}=\mathbb{P} \text { [at least one same subcarrier is used by at least two of the } K \text { tiers }\right] \\
& =1-\mathbb{P}[\text { No subcarrier is used simultaneously by } K \text { tiers }] \\
& =1-\prod_{j=1}^{K} \mathbb{P}\left[\text { No subcarrier is used simultaneously from the } i^{\text {th }} \text { tier }\right] \\
& =1-\prod_{j=1}^{K}\left(1-\frac{1}{M_{i}}\right)^{M_{j}}
\end{aligned}
$$

Thus, the interfering station in the $i^{t h}$ tier follows an i.m.p.p $\Phi_{i}$ with the intensity measure $\xi_{i}$, given as:

$$
\xi_{i}=p_{\text {coll }_{i}} \lambda_{i}
$$

The proof results from the overlapped resources assumption and from the thinning propriety of the Poisson Point Process (PPP) [21].

Let us denote $p_{\text {rbc }}$ as the probability of the event "At least one beacon is received from all tiers." which is derived as follows:

$$
\begin{aligned}
& p_{\mathrm{rbc}}=\mathbb{P}[\text { At least one beacon is received from all tiers }] \\
& =1-\mathbb{P}[\text { No beacons are received from the } k \text { tiers }] \\
& =1-\prod_{i=1}^{K}\left[\mathbb{P}\left[\text { No beacons are received from the } i^{t h} \text { tiers }\right]\right. \\
& =1-\prod_{i=1}^{K}(1-\zeta)^{N_{i}}
\end{aligned}
$$


Therefore, the received beacons from the $i^{\text {th }}$ tier follow an i.m.p.p $\Phi_{i}^{\prime}$ with the intensity measure $\Xi_{i}$, given as:

$$
\Xi_{i}=p_{\mathrm{rbc}} \zeta \lambda_{i}
$$

Finally, a deduction from the previous equation conduct to express the CCDF as follows:

$$
p_{\mathrm{cvg}}=\frac{\pi p_{\mathrm{rbc}} \zeta}{G(\alpha)} \frac{\sum_{i=1}^{K} \lambda_{i}\left(\frac{\widetilde{S I N R_{i}}}{P_{i}}\right)^{\frac{-2}{\alpha}}}{\sum_{i=1}^{K} p_{\operatorname{coll}_{i}} \lambda_{i} P_{i}^{\frac{2}{\alpha}}}
$$

where

$$
G(\alpha)=\frac{2 \pi^{2}}{\alpha \sin \left(\frac{2 \pi}{\alpha}\right)}
$$

To proved this results, we start by calculated:

$$
\begin{aligned}
p_{\mathrm{cvg}} & =\mathbb{P}\left[\bigcup_{i \in K, x_{j} \in \Phi_{i}^{\prime}} S I N R_{x_{j}}>\widehat{S I N R_{i}}\right] \\
& =\mathbb{E}\left[\mathbb{1} \bigcup_{i \in K, x_{j} \in \Phi_{i}^{\prime}} S I N R_{x_{j}}>\widehat{S I N R_{i}}\right] \\
& =\sum_{i \in K} \mathbb{E} \sum_{x_{j} \in \Phi_{i}^{\prime}}\left[\mathbb{1}\left(S I N R_{x_{j}}>\widehat{S I N R_{i}}\right)\right] \\
& =\sum_{i \in K} \Xi_{i} \int_{\mathbb{R}^{2}} \mathbb{P}\left(S I N R_{x_{j}}>\widehat{S I N R_{i}}\right) d x \\
& =\sum_{i \in K} \Xi_{i} \int_{\mathbb{R}^{2}}\left(1-\mathbb{P}\left(\widehat{S I N R_{x_{j}}} \leq \widehat{S I N R_{i}}\right)\right) d x \\
& =\sum_{i \in K} \Xi_{i} \int_{\mathbb{R}^{2}} \mathcal{L}_{I_{x_{j}}}\left(\frac{x^{\alpha} \widehat{S I N R_{i}}}{P_{i}}\right) d x
\end{aligned}
$$

where (11) yields from the sum of the probabilities of mutually exclusive events resulting from the assumption $\widetilde{S I N R}_{i}>1(0 d B),(12)$ follows the Campbell Mecke theorem [21] and (14) results from the expression of the outage probability given in?? where $\mathcal{L}_{I_{x_{j}}}$ is the Laplace transform of the cumulative interference from all the tiers when the typical user is being served by the $i^{t h}$ tier. Therefore,

$$
\begin{aligned}
\mathcal{L}_{I_{x_{j}}}(s) & =\prod_{i=1}^{K} \mathbb{E}_{\Phi_{i}, h}\left[\exp \left(-s \sum_{k=1, k \neq i} P_{k} h x_{k}^{-\alpha}\right)\right] \\
& =\prod_{i=1}^{K} \mathbb{E}_{\Phi_{i}}\left[\prod_{x_{k} \in \phi_{i} / x_{j}} \mathbb{E}_{h}\left[\exp \left(-s P_{k} h x_{k}^{-\alpha}\right)\right]\right] \\
& =\prod_{i=1}^{K} \exp \left[-\xi_{i} \int_{o}^{i n f t y}\left(1-\mathbb{E}_{h}\left[\exp \left(-s P_{i} h x_{i}^{-\alpha}\right)\right]\right) d x\right] \\
& =\prod_{i=1}^{K} \exp \left[-\xi_{i} \int_{o}^{i n f t y}\left(1-\frac{1}{1+s P_{i} h x_{i}^{-\alpha}}\right) d x\right] \\
& =\exp \left[-s^{\frac{2}{\alpha}} G(\alpha) \sum_{i=1}^{K} \xi_{i} P_{i}^{2 / \alpha}\right]
\end{aligned}
$$


where (16) results from the assumption of the independence between the fading and the PPP which yields to move the expectation over $h$ inside the product, and (17) follows the generating functional [21] for the PPP. A simple deduction leads to:

$$
\begin{aligned}
& p_{\mathrm{cvg}}=\sum_{i \in K} \Xi_{i} \int_{\mathbb{R}^{2}}\left[1-\mathbb{P}\left(\operatorname{SIN} R_{x} \leq \widetilde{\operatorname{SINR}} i\right)\right] d x \\
& =\sum_{i \in K} \Xi_{i} \int_{\mathbb{R}^{2}} \exp \left[-\left(\widetilde{\frac{\operatorname{SINR}_{i}}{P_{i}}}\right)^{\frac{2}{\alpha}} G(\alpha) x_{i}^{2} \sum_{k=1}^{K} \xi_{k} P_{k}^{\frac{2}{\alpha}}\right] d x \\
& =\frac{\pi}{G(\alpha)} \frac{\sum_{i=1}^{K} \Xi_{i}\left(\frac{\widetilde{\operatorname{SINR}}}{P_{i}}\right)^{-2 / \alpha}}{\sum_{i=1}^{K} \xi_{i} P_{i}^{\frac{2}{\alpha}}} \\
& =\frac{\pi p_{\mathrm{rbc}} \zeta}{G(\alpha)} \frac{\sum_{i=1}^{K} \lambda_{i}\left(\widetilde{\frac{\widetilde{S I N R}_{i}}{P_{i}}}\right)^{\frac{-2}{\alpha}}}{\sum_{i=1}^{K} p_{\mathrm{coll}_{i}} \lambda_{i} P_{i}^{\frac{2}{\alpha}}}
\end{aligned}
$$
by:

Taking the simplest case where $\widetilde{\operatorname{SINR}_{i}}=\widetilde{\operatorname{SINR}}, \forall i \in\{K\}$, we observe that the coverage probability is given

$$
p_{\mathrm{cvg}}=\frac{\pi p_{\mathrm{rbc}} \zeta}{G(\alpha)} \widetilde{S I N R} \frac{\sum_{i=1}^{K} \lambda_{i} P_{i}^{\frac{2}{\alpha}}}{\sum_{i=1}^{K} p_{\mathrm{coll}_{i}} \lambda_{i} P_{i}^{\frac{2}{\alpha}}}
$$

which differs from the conventional model that only depends on the SINR threshold $\widetilde{S I N R}$. It is clear that the

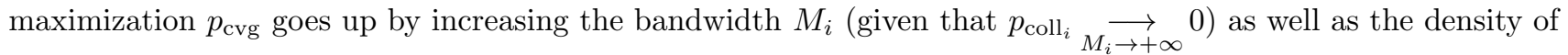
stations in the tiers (given that $p_{\mathrm{rbc}} \underset{\lambda_{i} \rightarrow+\infty}{\longrightarrow} 1$ ).

\section{Energy efficiency}

The energy efficiency was presented by the ratio between the area's spectral network and the average network power consumption, as $\Theta_{E E}$, given by:

$$
\Theta_{E E}=\frac{\text { Area spectral efficiency }}{\text { Total network area power consumption }}
$$

where the area spectral efficiency defined as the sum of the average throughput is given as follows:

$$
R=p_{\operatorname{cvg}} \sum_{i=1}^{K} B \log \left(1+\widetilde{S I N R_{i}}\right)
$$

and, the total consumption power $P_{T}$ is expressed by:

$$
P_{T}=\sum_{i=1}^{K} \lambda_{i}\left(\frac{1}{\beta_{i}} P_{R S_{i}}+P_{S S_{i}}\right)
$$

where $P_{R S_{i}}$ and $P_{S S_{i}}$ are the $i^{\text {th }}$ SCS radiated power and static power, respectively, and $\beta_{i}$ is the $i^{\text {th }}$ SCS power amplifier efficiency.

Based on these results, the following EE formula is conducted:

$$
\Theta_{E E}=\frac{p_{c v g} \sum_{i=1}^{K} B \log \left(1+\widetilde{S I N R_{i}}\right)}{\sum_{i=1}^{K} \lambda_{i}\left(\frac{1}{\beta_{i}} P_{R S_{i}}+P_{S S_{i}}\right)}
$$




\section{Results and discussion}

In a first step, in terms of coverage probability, the analyses are shown based on the simulation results. After that, the difference between the suggested DCCMC scheme and the conventional model, which is subject to a severe ICI, is illustrated. The performance of the proposed protocol is proved compared to the eICIC, which is based on the ABS concept. As a second step, in terms of energy efficiency, the performances of the suggested scheme in a three-tier network are proved compared to the imperfect sensing SCS model.

To this end, we first, consider three different network deployments as follows: 2-tier, 3-tier and 4-tier located over an approximately zone with an area $A>\sum\left(A_{i}\right)$. Each tier has different SCSs that follow an independent PPP with the density measure $\lambda_{i}$, respective to the $i^{\text {th }}$ tier and assuming that $\lambda_{i}=i \lambda, i \in\{2,4\}$. To facilitate the analysis, we take the case where the considered tiers have the same $S I N R$ threshold $\widetilde{S I N R}$, which is greater than $1(0 \mathrm{~dB})$. In addition, we treat the case where $\alpha=3.2$, the noise power $W=0$, and the probability $\zeta=0.3$.

As shown in Figure 1 which depicts the coverage probability considering different case network deployments (2-tier, 3-tier and 4-tier) for both proposed and conventional scenarios, the DCCMC scheme scenario with 4 tiers yields the most optimistic coverage probability $p_{\text {cvg }}$. This result matches reasonably with the analysis results. Hence, it is clearly observed that the coverage probability goes up resulting on the interference decrease when the number of tiers rise, unlike the conventional model that depends neither on the number of tiers nor on the number of cell-stations because it is similar to a single tier with a fixed threshold $\widetilde{S I N R}$. Obviously, the coverage probability under the DCCMC approach increases with the rise of the cell-station density, until reaching a constant optimized $p_{\text {cvg }}$ value. However, for a small cell station density, applying the DCCMC methods seems not reliable, which result from the rise of the collision probability.

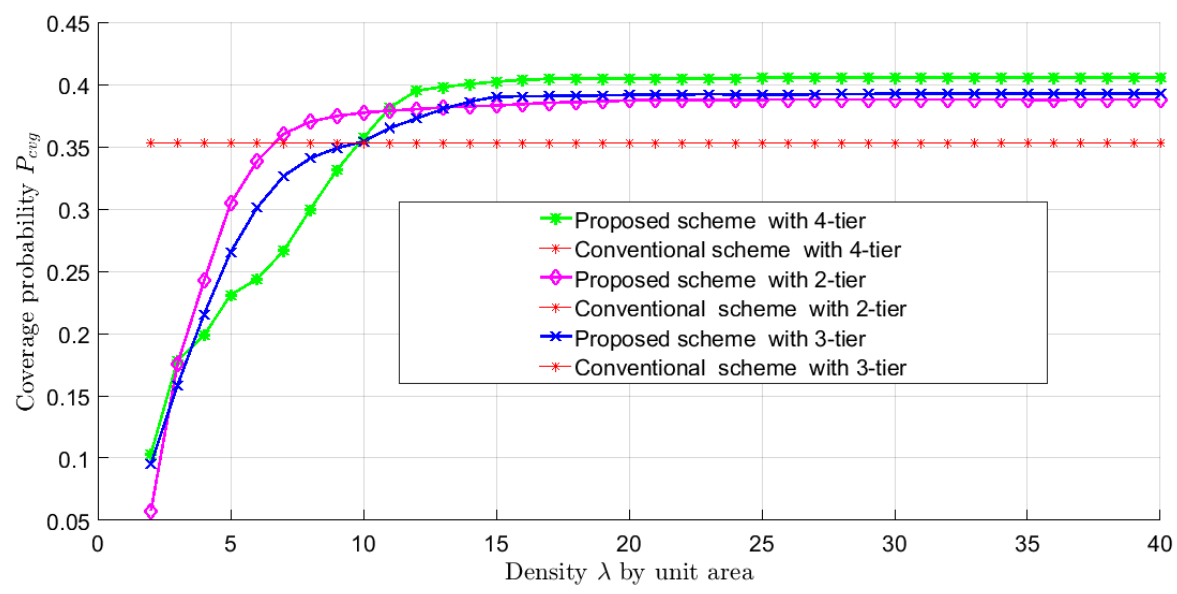

Fig. 1. Coverage probability vs SCSs density with 2, 3 and 4 tiers

Furthermore, Figure 2 illustrates the performance in terms of coverage probability under various numbers of $S I N R$ threshold $\widetilde{S I N R}$. A significant performance improvement in terms of coverage probability, relative to the DCCMC scheme for all the mentioned cases (2 tier, 3 tier and 4 tier) can be observed compared to the conventional model. Unlike the conventional scenario that does not depend on the number of tiers, the same remark regarding the growth of coverage probability with the increase in the number of tiers is noticed. Indeed, when the number of tiers goes up the collision probability goes down yielding on the reduction of the amount of interference.

The case of 2-tier heterogenous network deployment, where the tier of the SSs is overlaid under the macro base station tier, the classical enhanced ICIC is compared with the proposed DCCMC concept, in terms of coverage probability, as in Figure 3. Obviously, the DCCMC protocol may be more beneficial than the eICIC approach which is based on the ABS technique. This is because the intra cell interference between SCSs is not taken into account in the eICIC. Therefore, the DCCMC, can achieves as better performance given that the cell-edge users will not suffer from coverage degradation thanks to the use of the virtual coordination based on the cognitive monitoring strategy.

As a second step, in order to depict the performance of the suggested DCCMC protocol compared to other models, in terms of energy efficiency, a three-tier network is considered.Figure 4, shows the impact of the SCSs 


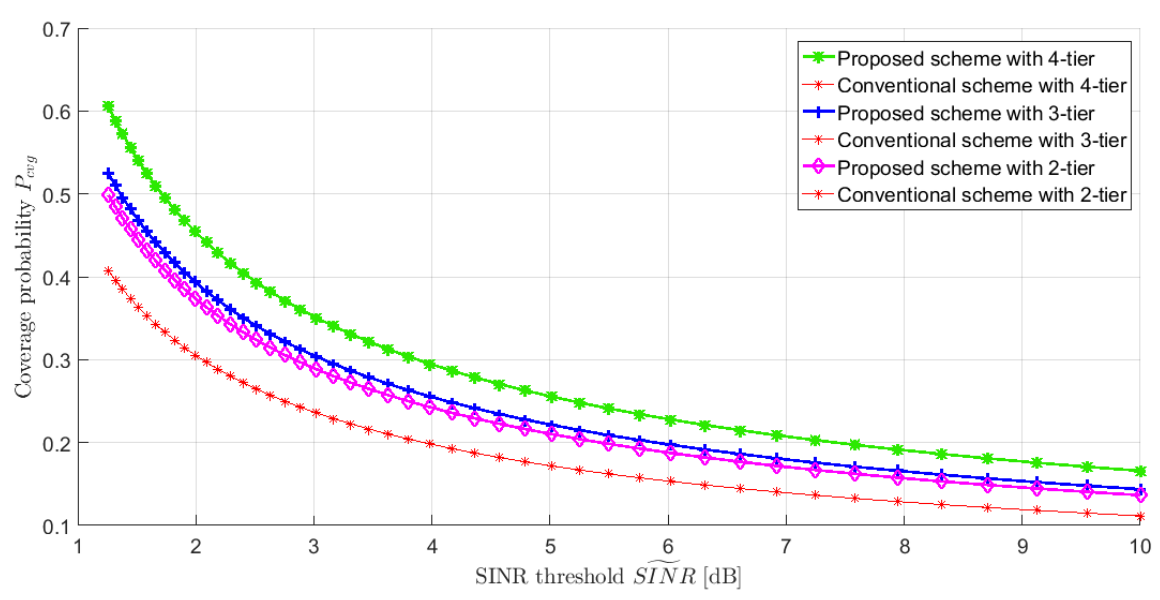

Fig. 2. Coverage probability vs $\widetilde{S I N R}$ with with 2,3 and 4 tiers

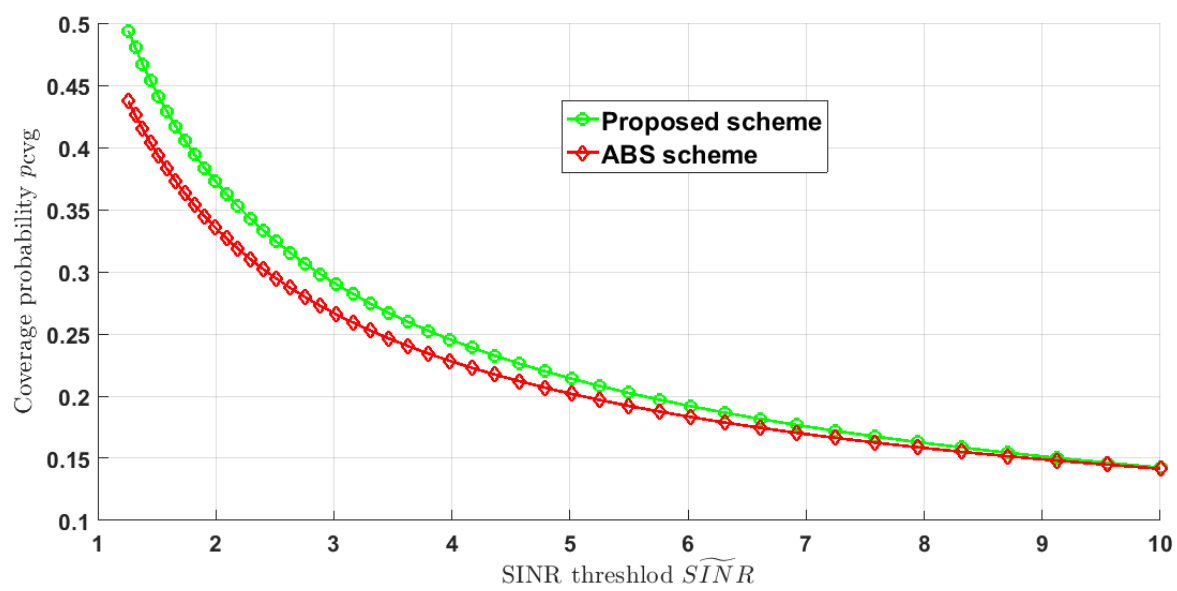

Fig. 3. Coverage probability vs $\widetilde{S I N R}$ with 2 tiers

density $\lambda$ on the energy efficiency metric and compares the performance of the suggested model with the SCSs imperfect sensing one. For a lower density value, as the density increases, the EE goes up until achieving a maximum value for an optimal SCS density. Therefore, for a density greater than the optimal value, even if the number of SCSs rises the EE remains constant. Thus, there exist a limit for the network densification and an optimal SCS density that should be defined in order to achieve the maximum EE with the minimum deployment costs. Most importantly, the EE relative to the proposed model outperforms that of imperfect SCS sensing one. In fact, about $58 \%$ of gain performance is noticed.

Figure 5, presents the performance gains of the suggested model, in terms of EE, under various SINR thresholds $\widehat{S I N R}$. The SCS density $\lambda$ is equal to $0.6 \times 10^{-4} / \mathrm{m}^{2}$. For a low value of $\widehat{S I N R}$, the EE performance - with the increasing of $\widehat{S I N R}$ - grows until reaching an optimal value. However, for a larger value of $\widehat{S I N R}$, the EE declines. Clearly, the performances of the suggested framework are proved compared to the imperfect SCS sensing scenario. About $66 \%$ of again performance is noticed compared to the other scenarios.

\section{Conclusion}

This paper proposes a novel approach to manage the interference in ultra-dense heterogeneous cellular networks. The suggested DCCMC protocol is modeled and developed, based on the stochastic geometry model. Under some general assumptions, the resulting expressions for coverage probability as well energy efficiency are involved as a closed-form expression. The numerical results are illustrated, proving the analysis and improving the performance of the proposed framework compared to the conventional model and the eICIC one as well as the SCSs imperfect sensing scenario. However, it is proved that an optimal number of deployed SCSs is necessary to achieve a 


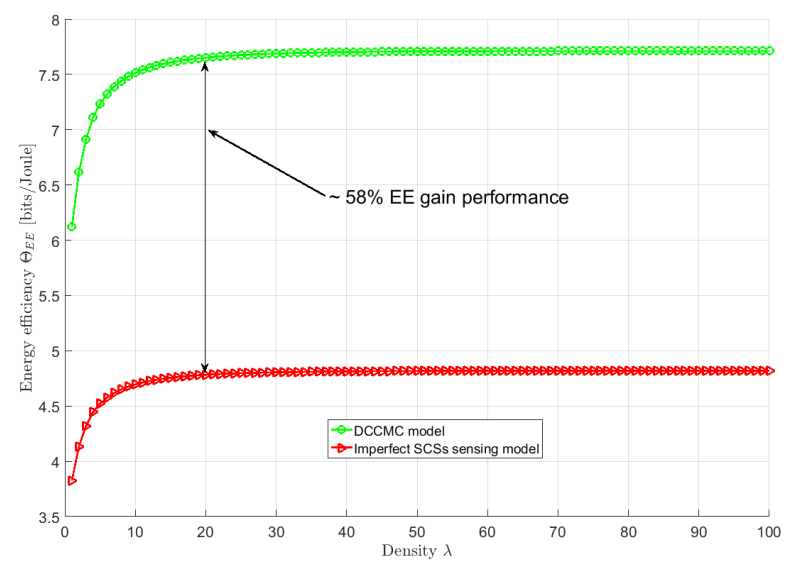

Fig. 4. Energy efficiency vs SCSs density $\lambda$

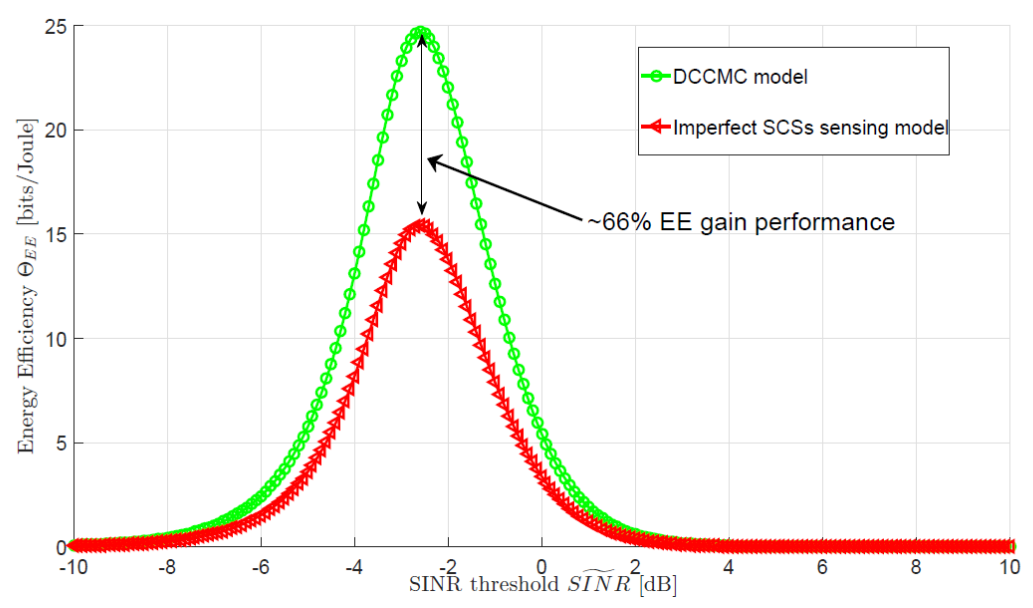

Fig. 5. Energy efficiency vs $\widetilde{S I N R}$

maximum energy efficiency network design. Thus, densifying the network with a number of SCSs beyond this optimal value will not improve the energy efficiency performances in an UDHetNet.

\section{References}

1. O. Blume, D. Zeller, and U. Barth, "Approaches to energy efficient wireless access networks," in Communications, Control and Signal Processing (ISCCSP), 2010 4th International Symposium on, pp. 1-5, IEEE, 2010.

2. M. Webb, "Smart 2020: enabling the low carbon economy in the information age, a report by the climate group on behalf of the global esustainability initiative (gesi)," Global eSustainability Initiative (GeSI), Tech. Rep , 2008.

3. M. Gruber, O. Blume, D. Ferling, D. Zeller, M. A. Imran, and E. C. Strinati, "Earth-energy aware radio and network technologies," in Personal, Indoor and Mobile Radio Communications, 2009 IEEE 20th International Symposium on, pp. 1-5, IEEE, 2009.

4. I. S. Outlook, "An assessment of the current state of affairs and a path towards improved sustainability for public policies," Report, BIO Intelligence Service and Alcatel-Lucent, 2013.

5. F. Richter, A. J. Fehske, and G. P. Fettweis, "Energy efficiency aspects of base station deployment strategies for cellular networks," in Vehicular Technology Conference Fall (VTC 2009-Fall), 2009 IEEE 70th, pp. 1-5, IEEE, 2009.

6. E. Oh, B. Krishnamachari, X. Liu, and Z. Niu, "Toward dynamic energy-efficient operation of cellular network infrastructure," IEEE Communications Magazine 49(6), 2011.

7. G. Fettweis and E. Zimmermann, "Ict energy consumption-trends and challenges," in Proceedings of the 11th international symposium on wireless personal multimedia communications, 2(4), p. 6, (Lapland, 2008.

8. L. Su, C. Yang, Z. Xu, and A. F. Molisch, "Energy-efficient downlink transmission with base station closing in small cell networks," in Acoustics, Speech and Signal Processing (ICASSP), 2013 IEEE International Conference on, pp. 4784-4788, IEEE, 2013. 
9. Y. Lin, W. Bao, W. Yu, and B. Liang, "Optimizing user association and spectrum allocation in hetnets: A utility perspective," IEEE Journal on Selected Areas in Communications 33(6), pp. 1025-1039, 2015.

10. W. Datasheet, "Lte release 8,"

11. M. Abdullah and A. Yonis, "Performance of lte release 8 and release 10 in wireless communications," in Cyber Security, Cyber Warfare and Digital Forensic (CyberSec), 2012 International Conference on, pp. 236-241, IEEE, 2012.

12. A. Damnjanovic, J. Montojo, Y. Wei, T. Ji, T. Luo, M. Vajapeyam, T. Yoo, O. Song, and D. Malladi, "A survey on 3gpp heterogeneous networks," IEEE Wireless Communications 18(3), 2011.

13. S. Deb, P. Monogioudis, J. Miernik, and J. P. Seymour, "Algorithms for enhanced inter-cell interference coordination (eicic) in lte hetnets," IEEE/ACM transactions on networking 22(1), pp. 137-150, 2014.

14. Y. Dhungana and C. Tellambura, "Multichannel analysis of cell range expansion and resource partitioning in two-tier heterogeneous cellular networks," IEEE Transactions on Wireless Communications 15(3), pp. 2394-2406, 2016.

15. R. Mendrzik, R. A. J. Castillo, G. Bauch, and E. Seidel, "Interference coordination-based downlink scheduling for heterogeneous lte-a networks," in Wireless Communications and Networking Conference (WCNC), 2016 IEEE, pp. 16, IEEE, 2016.

16. H. ElSawy and E. Hossain, "On cognitive small cells in two-tier heterogeneous networks," in Modeling E Optimization in Mobile, Ad Hoc \& Wireless Networks (WiOpt), 2013 11th International Symposium on, pp. 75-82, IEEE, 2013.

17. F. H. Panahi and T. Ohtsuki, "Stochastic geometry modeling and analysis of cognitive heterogeneous cellular networks," EURASIP Journal on Wireless Communications and Networking 2015(1), p. 141, 2015.

18. A. Bohli and R. Bouallegue, "Stochastic geometry model to analyze $5 \mathrm{~g}$ energy efficiency based on a novel dynamic spectrum access scheme," Transactions on Emerging Telecommunications Technologies 27(12), pp. 1715-1728, 2016.

19. A. Bohli and R. Bouallegue, "A new sensing strategy for $5 \mathrm{~g}$ mobile networks: Towards spectral and energy efficiency trade off," in Advanced Information Networking and Applications (AINA), 2016 IEEE 30th International Conference on, pp. 155-159, IEEE, 2016.

20. H. S. Dhillon, R. K. Ganti, F. Baccelli, and J. G. Andrews, "Modeling and analysis of k-tier downlink heterogeneous cellular networks," IEEE Journal on Selected Areas in Communications 30(3), pp. 550-560, 2012.

21. F. Baccelli, B. Błaszczyszyn, et al., "Stochastic geometry and wireless networks: Volume ii applications," Foundations and Trends® in Networking 4(1-2), pp. 1-312, 2010.

22. C. Jedrzycki and V. C. Leung, "Probability distribution of channel holding time in cellular telephony systems," in Vehicular Technology Conference, 1996. Mobile Technology for the Human Race., IEEE 46th, 1, pp. 247-251, IEEE, 1996. 